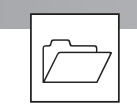

Endokrynologia Polska

DOI: 10.5603/EP.a2021.0025

Volume/Tom 72; Number/Numer 3/2021

ISSN 0423-104X, e-ISSN 2299-8306

\title{
Clinicopathological features of neonatal subcutaneous fat necrosis and its implications
}

\author{
Cristina Ferreras ${ }^{1,2}$, Vanessa Gorito ${ }^{1,2}$, Ana Vilan ${ }^{2,3}$, Jorge Pedro ${ }^{4}$, Rita Santos Silva ${ }^{2,5}$, \\ Cintia Castro-Correia ${ }^{2,5}$, Carla Costa ${ }^{2,5}$, Henrique Soares ${ }^{2,3}$ \\ ${ }^{1}$ Department of Paediatrics, Centro Hospitalar São João, Porto, Portugal \\ ${ }^{2}$ Faculty of Medicine, University of Porto, Portugal \\ ${ }^{3}$ Department of Neonatology, Centro Hospitalar São João, Porto, Portugal \\ ${ }^{4}$ Department of Endocrinology, Centro Hospitalar São João, Porto, Portugal \\ ${ }^{5}$ Pediatric Endocrinology and Diabetology Unit, Department of Paediatrics, Centro Hospitalar São João, Porto, Portugal
}

Key words: subcutaneous fat necrosis; newborn; hypercalcaemia

\section{Case 1}

A full-term infant girl was delivered by elective caesarean section at $38+3$ gestational weeks. Pregnancy history was unremarkable. Birth weight: 3465 grams. Apgar score was 9, 10, and 10 at 1,5 , and 10 min, respectively. There were no complications in the immediate neonatal period. On the fifth day of life, an erythematous, hard, and painless lesion $(2 \times 2 \mathrm{~cm})$ in the right upper limb was detected (Fig. 1). Ultrasound revealed thickening and hyperechogenicity of the subcutaneous cellular tissue, compatible with subcutaneous fat necrosis (SCFN). Initial laboratorial studies were unremarkable, except mildly increased ionized calcium $5.53 \mathrm{mg} / \mathrm{dL}$ (reference range: $4.8-5.52 \mathrm{mg} / \mathrm{dL}$ ). The patient did not receive vitamin D supplements. Subsequent laboratorial studies revealed no changes. There was spontaneous

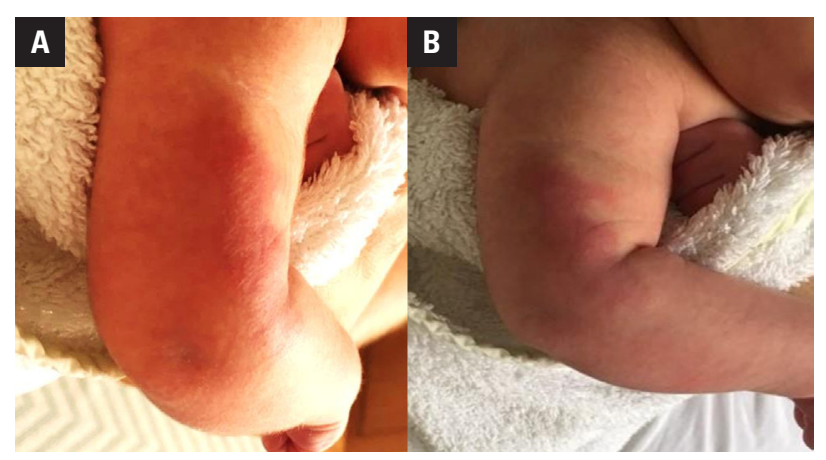

Figure 1AB. Subcutaneous fat necrosis (SCFN) hard nodules covered by erythematous skin, localized in the right arm progressive reduction of the lesion and complete disappearance at 6 months.

\section{Case 2}

A female term newborn was delivered by elective caesarean section at 38 weeks following an uncomplicated pregnancy. Birth weight: $3700 \mathrm{~g}$. Apgar score was 10,10, and 10 at 1, 5, and $10 \mathrm{~min}$, respectively. At birth, she had an erythematous, hard, and painless lesion in the left upper limb $(3 \times 3 \mathrm{~cm})$ (Fig. 2). Ultrasound showed heterogeneous and hyperechoic thickening of the subcutaneous cell tissue, with 2-mm cystic areas compatible with SCFN. Before discharge, blood tests performed showed mild hypercalcaemia (calcium $11.6 \mathrm{mg} / \mathrm{dL}$; reference $7.6-10.4 \mathrm{mg} / \mathrm{dL}$ ) and decreased parathyroid hormone $(9.9 \mathrm{pg} / \mathrm{mL}$; reference $15-65$

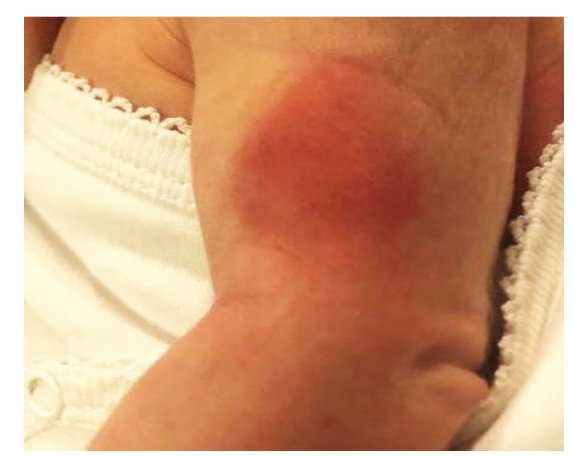

Figure 2. Subcutaneous fat necrosis (SCFN) localized on the left arm 
$\mathrm{pg} / \mathrm{mL}$ ). She was not supplemented with vitamin D. During follow-up she maintained hypercalcaemia $(10.8 \mathrm{mg} / \mathrm{dL})$ and was referred to the Paediatric Endocrinology Clinic for evaluation. At 5 months of age, calcium and 25-OH-vitamin D values were normal, and the lesion decreased. At 9 months, the baby was in good clinical condition and the lesion disappeared.

Subcutaneous fat necrosis of the newborn is an uncommon disorder, with self-limiting panniculitis presenting in the first few weeks after birth as wide erythematous plaques or firm non-tender subcutaneous nodules, which most commonly affect the limbs, buttocks, back, cheeks, and areas over bony prominences $[1,2]$.

The aetiology and pathogenesis of SCFN is unknown. It is postulated that subcutaneous tissue hypoperfusion aggravates the deposition of saturated fatty acids, and because the system enzymes are immature there is increasing deposition of saturated fatty acids, leading to adipose tissue solidification and necrosis. The local pressure resulting from childbirth trauma can increase the tendency to fat necrosis [3].

The diagnosis is clinical, suggested by history and characteristic clinical findings. Imaging exams are generally unnecessary for diagnosis, although they play an important role in excluding other causes because the presence of soft tissue swelling in newborns requires a detailed investigation [5]. Skin biopsy is not necessary to establish the diagnosis, especially if there are no concerns about the differential diagnosis. In our 2 cases, clinical evaluation together with non-invasive imaging were essential for timely recognition, without the need for biopsy.

The most important differential diagnosis is sclerema neonatorum, a disorder in which there is a thickening of the skin secondary to fibrosis of the trabecular septa of the adipocytes' involvement of the muscle and bone tissue, associated with congenital malformations, cyanosis, respiratory disorders, and sepsis [3]. Another differential diagnosis is scleredema, which presents progressive oedema of the extremities and stiffening of the affected limbs, usually preceded by gastrointestinal or respiratory infections; pathology reveals intense interstitial oedema associated with lobular panniculitis without vasculitis. Other differential diagnosis includes skin infections, CMV infection, haemangiomas, Farber disease, and sarcomas [1, 3].

This is a benign condition which usually resolves spontaneously in less than a year. However, it can be accompanied by extracutaneous changes, namely hypoglycaemia, anaemia, thrombocytopaenia, and hypercalcaemia.
Hypercalcaemia is the main complication of this pathology; it is found in $25-50 \%$ of the cases and may be present before the subcutaneous nodules appear. In a systematic review of published cases of SCFN, hypercalcaemia was detected in $57 \%$ in the first 28 days of life and in $30 \%$ in the following 28 days. This complication appears 1-6 months after SCFN, and therefore monitoring serum calcium levels is essential. Since regression of the lesions is usually associated with an increase in serum calcium levels, some authors recommend monitoring them at 30,45, and 60 days after the disappearance of skin lesions [1]. The pathogenesis of the hypercalcaemia is also not understood. Granulomatous inflammatory cells in SCFN express high levels of 1-alpha-hydroxylase, the enzyme that converts 25-hydroxyvitamin D3 to its active form of 1,25-dihydroxyvitamin D3. 1,25-dihydroxyvitamin D3 promotes calcium mobilization from bone and increases intestinal absorption of calcium. Therefore, it is plausible that increased 1,25-dihydroxyvitamin D3 production in affected tissue of SCFN results in secondary hypercalcaemia [4]. Increased prostaglandin E or parathyroid hormone levels have been postulated; however, they are often normal in these infants [5]. Additionally, it has been suggested that decreased renal clearance of calcium may contribute to SCFN-associated hypercalcaemia. These changes are important since failure to implement prompt therapy, including restriction of supplemental calcium and vitamin $\mathrm{D}$, hyperhydration with intravenous fluids, and calcium-wasting loop diuretics can lead to severe complications such as nephrolithiasis and acute renal injury. In more severe cases, systemic corticosteroids and bisphosphonates have been used. When SCFN is not associated with hypercalcaemia, the treatment of this dermatosis is symptomatic, requiring the avoidance of vitamin D supplementation [1-3].

\section{References}

1. Stefanko NS, Drolet BA. Subcutaneous fat necrosis of the newborn and associated hypercalcemia: A systematic review of the literature. Pediatr Dermatol. 2019; 36(1): 24-30, doi: 10.1111/pde.13640, indexed in Pubmed: 30187956

2. Kannenberg SMH, Jordaan HF, Visser WI, et al. Report of 2 Nove Presentations of Subcutaneous Fat Necrosis of the Newborn. Dermatopathology (Basel). 2019; 6(2): 147-152, doi: 10.1159/000497176, indexed in Pubmed: 31700855.

3. Muzy G, Mayor SA, Lellis RF. Subcutaneous fat necrosis of the newborn: clinical and histopathological correlation. An Bras Dermatol. 2018; 93(3): 412-414, doi: 10.1590/abd1806-4841.20187508, indexed in Pubmed: 29924224

4. Farooque A, Moss C, Zehnder D, et al. Expression of 25-hydroxyvitamin D3-1alpha-hydroxylase in subcutaneous fat necrosis. Br J Dermatol. 2009; 160(2): 423-425, doi: 10.1111/j.1365-2133.2008.08844.x, indexed in Pubmed: 18811689

5. Sharata H, Postellon DC, Hashimoto K. Subcutaneous fat necrosis, hypercalcemia, and prostaglandin E. Pediatr Dermatol. 1995; 12(1): 43-47, doi: 10.1111/j.1525-1470.1995.tb00123.x, indexed in Pubmed: 7792219. 